\title{
Detecting CDOM Fluorescence Using High Spectrally Resolved Satellite Data: A Model Study
}

\author{
Aleksandra Wolanin, Vladimir Rozanov, Tilman Dinter \\ and Astrid Bracher
}

\begin{abstract}
Absorption and fluorescence of CDOM are widely used to characterize its concentration and composition, both in situ and with remote sensing techniques. As fluorescence is an inelastic scattering process, it can potentially be observed in filling-in of Fraunhofer lines with the DOAS method in high spectrally resolved satellite data. Here, we perform a theoretical model study and preliminary DOAS retrieval on SCIAMACHY data in order to test the feasibility of such an approach. Our results show that retrieving CDOM fluorescence is difficult, due to its relatively weak signal in global oceans (in comparison to Raman scattering of clear water) and varying broad spectral features. Detailed studies of the synergistic use of narrow and broad fluorescence features, and an appropriate scheme to account for disturbance effects of Raman scattering, are needed for further investigating the retrieval of CDOM fluorescence.
\end{abstract}

\section{Introduction}

Colored or chromophoric dissolved organic matter (CDOM) is part of the dissolved organic matter (DOM) pool, which significantly absorbs light in the UV and blue region of the light spectrum. CDOM plays a role in many physical, chemical and biological processes in aquatic systems. Primarily, it reduces potentially harmful UV radiation within the water column, but on the other hand-when abundantlimits the amount of light available for photosynthesis.

Optical parameters of CDOM are widely used to characterize it both qualitatively and quantitatively. Absorbance and fluorescence of bulks of CDOM are measured

\footnotetext{
A. Wolanin $(\bowtie) \cdot$ V. Rozanov $\cdot$ T. Dinter $\cdot$ A. Bracher

Institute of Environmental Physics, University of Bremen, Bremen, Germany

e-mail: ola@iup.physik.uni-bremen.de
}

\author{
A. Wolanin · A. Bracher \\ Alfred Wegener Institute Helmholtz Centre for Polar and Marine Research, \\ Bremerhaven, Germany \\ (C) Springer International Publishing Switzerland 2015 \\ G. Lohmann et al. (eds.), Towards an Interdisciplinary Approach \\ in Earth System Science, Springer Earth System Sciences, \\ DOI 10.1007/978-3-319-13865-7_13
}


locally and globally to examine water quality, chemical composition of dissolved organic matter, trace water masses and study dynamical processes involving CDOM. $\mathrm{CDOM}$ fluorescence is a significant inelastic process in natural waters, along with vibrational Raman scattering (VRS) and chlorophyll fluorescence.

Between processes of absorption and emission some energy is dissipated, so the energy emitted is lower than the energy absorbed. The wavelengths at which a photon is absorbed and emitted depend mainly on the molecule itself, but can also depend on several environmental factors, e.g. $\mathrm{pH}$, temperature, a presence of certain metals (Hudson et al. 2007). Fluorescent CDOM is a complex mixture of many fluorescent compounds, and individual fluorophores are hard to identify.

Because of the shift of wavelength in the fluorescence process, its effect on the backscattered radiation at the top of atmosphere is possibly identified in the fillingin of Fraunhofer lines, which are spectrally narrow, often saturated absorption features in the solar spectrum. Other inelastic processes have been already retrieved from hyperspectral satellite data with the Differential Optical Absorption Spectroscopy (DOAS) method (e.g. Vountas et al. 1998, 2003, 2007). In this work we test the use of the filling-in signal originating from CDOM fluorescence to characterize CDOM composition and concentration independent from chlorophyll. This method would also help to improve chlorophyll retrievals in regions of high CDOM concentrations.

\section{Methods}

\subsection{DOAS Retrieval Technique}

Different variants of the DOAS technique have been applied for several years for the retrieval of atmospheric trace gases from satellite measurements (e.g. Burrows et al. 1999; Richter et al. 2005), and have lately been extended to the aquatic domain as PhytoDOAS (Vountas et al. 2007: Bracher et al. 2009; Sadeghi et al. 2012). The DOAS method is based on Beer-Lambert's law (e.g. Rozanov and Rozanov 2010) and determines the amount of molecular absorbers along the effective optical light path by fitting and scaling spectra within a given wavelength window. The broad-band effects (e.g. Rayleigh and Mie scattering) are removed by a low-degree polynomial that is also fitted. The fitting in the DOAS method is formalized as a least-squares minimization and is described by the following equation:

$$
\left\|\tau(\lambda)-\sum_{n=1}^{N} \sigma_{n}(\lambda) S_{n}-\sigma_{R}(\lambda) S_{R}-\sigma_{V}(\lambda) S_{V}-\sigma_{f}(\lambda) S_{f}-\sum_{m=0}^{M} a_{m} \lambda^{m}\right\|^{2} \rightarrow \text { min } .
$$

Here, $\tau(\lambda)=-\ln \frac{I(\lambda)}{I_{0}(\lambda)}$ is the so-called slant optical density, $I(\lambda)$ and $I_{0}(\lambda)$ are the measured backscattered radiance and extraterrestrial irradiance, respectively; 
$\sigma_{n}(\lambda)$ is the absorption cross-section of the $n$th atmospheric absorber; $N$ is the number of absorbers; $\sigma_{R}(\lambda), \sigma_{V}(\lambda)$, and $\sigma_{f}(\lambda)$ are reference spectra of rotational Raman scattering (RRS), VRS and CDOM fluorescence, respectively; $\sum_{m=0}^{M} a_{m} \lambda^{m}$ is the low order polynomial, typically of the order $M \leq 4$.

The minimization is carried out with $S_{n}, S_{R}, S_{V}, S_{f}$ and polynomial coefficients as the fitted parameters. The parameter $S_{n}$ is the integrated number density of the $n$th atmospheric absorber along the slant optical path, which is generally related to the concentration of a given absorber. Parameters $S_{R}, S_{V}$, and $S_{f}$ are called scaling or fit factors and carry information about the strength of the corresponding inelastic process.

\subsection{CDOM Fluorescence Modeling}

In order to perform the CDOM fluorescence retrieval with the DOAS method, an adequate fluorescence spectrum has to be used as a priori information, which is then scaled in the fitting procedure. The reference spectrum for CDOM fluorescence is calculated as the difference between the optical density modeled without and with CDOM fluorescence (after Vountas et al. 1998):

$$
\sigma_{f}(\lambda)=\ln \frac{I^{+}(\lambda)}{I^{-}(\lambda)}
$$

where $I^{+}(\lambda)$ and $I^{-}(\lambda)$ are modeled radiances calculated including and excluding inelastic processes, respectively.

The radiances $I^{+}(\lambda)$ and $I^{-}(\lambda)$ at the top of the atmosphere have been calculated by using the coupled ocean-atmosphere radiative transfer model SCIATRAN (Rozanov et al. 2014; Blum et al. 2012). Atmospheric conditions, namely the pressure, temperature, and water vapor profiles were set as default in the model for April and latitude of $45^{\circ}$, according to a climatological database obtained using a 2D chemical transport model developed at the University of Bremen (Sinnhuber et al. 2009). The solar zenith angle was set to $45^{\circ}$. Fluorescence was implemented using the spectral volume inelastic function $\beta\left(z ; \xi^{\prime} \rightarrow \xi ; \lambda^{\prime} \rightarrow \lambda\right)$, after Mobley (1994):

$$
\beta\left(z ; \xi^{\prime} \rightarrow \xi ; \lambda^{\prime} \rightarrow \lambda\right)=a\left(z ; \lambda^{\prime}\right) R\left(\lambda^{\prime} \rightarrow \lambda\right) \tilde{\beta}\left(\xi^{\prime} \rightarrow \xi\right)\left[m^{-1} s r^{-1} n m-1\right],
$$

where $a\left(z ; \lambda^{\prime}\right)$ is an absorption coefficient at the depth $\mathrm{z}$ and wavelength $\lambda^{\prime}$, usually expressed as a product of a specific absorption coefficient $a^{*}\left(\lambda^{\prime}\right)$ and concentration $C(z) \cdot R\left(\lambda^{\prime} \rightarrow \lambda\right)$ is a wavelength redistribution function from excitation wavelength $\lambda^{\prime}$ to emission wavelength $\lambda . \tilde{\beta}\left(\xi^{\prime} \rightarrow \xi\right)$ is a phase function for the radiance emitted from a direction $\xi^{\prime}$ to a direction $\xi$, which is assumed to be isotropic. The CDOM fluorescence was implemented into the SCIATRAN radiative transfer model on the base of two different approaches as described below. 


\subsubsection{CDOM as Mixture of Humic and Fulvic Acids}

In his work, Hawes (1992) assumed that fulvic and humic acids comprise the sum of total CDOM (although it is not usually true). Hawes (1992) isolated humic and fulvic acids from several samples and measured their absorption and fluorescence; $a^{*}\left(\lambda^{\prime}\right)$ and spectral fluorescence quantum efficiency functions $\eta\left(\lambda^{\prime} \rightarrow \lambda\right)$ were converted into mathematical functions with linear regression in order to use the data in optical models. He used $\eta\left(\lambda^{\prime} \rightarrow \lambda\right)$ (formulated in numbers of photons), from which we obtained $R\left(\lambda^{\prime} \rightarrow \lambda\right)$ (formulated in terms of energy), according to Mobley (1994). For the purpose of our study, absorption and fluorescence parameters of samples numbered FA7 (of fulvic acids) and HA6 (of humic acids) from Hawes (1992) were used. Specific absorption spectra and fluorescence quantum efficiency functions for these components are shown in Fig. 1. For the investigation of fluorescence structures in the whole spectral range, concentrations of fulvic and humic acids were set separately to 1 and $10 \mathrm{~g} \mathrm{~m}^{-3}$ in the $100 \mathrm{~m}$ deep water column. For narrow-band modeling in the spectral region of Fraunhofer line $\mathrm{H} \beta$ at $486 \mathrm{~nm}$ (see Sect. 3) concentrations of fulvic and humic acids were set simultaneously to $1,10,50$ and $100 \mathrm{~g} \mathrm{~m}^{-3}$.

CDOM abundance is typically quantified by its absorption coefficient at a given wavelength (e.g. 443 or $355 \mathrm{~nm})$. For most of the ocean $a(443 \mathrm{~nm})$ is below $0.1 \mathrm{~m}^{-1}$ (Morel and Gentili 2009), but can be much higher for coastal waters. In European coastal waters $a(443 \mathrm{~nm})$ ranges approximately between 0.01 and $1 \mathrm{~m}^{-1}$ (Babin, 2003). In our case, $1 \mathrm{~g} \mathrm{~m}^{-3}$ of fulvic or humic acids corresponds to $a(443 \mathrm{~nm})$ of $0.075 \mathrm{~m}^{-1}$ for FA7 and $0.011 \mathrm{~m}^{-1}$ for HA6.

\subsubsection{Excitation-Emission Matrices Approach}

In the second approach of implementing CDOM fluorescence into SCIATRAN we used a PARAFAC (PARAllel FACtor analysis) model. Nowadays, PARAFAC is
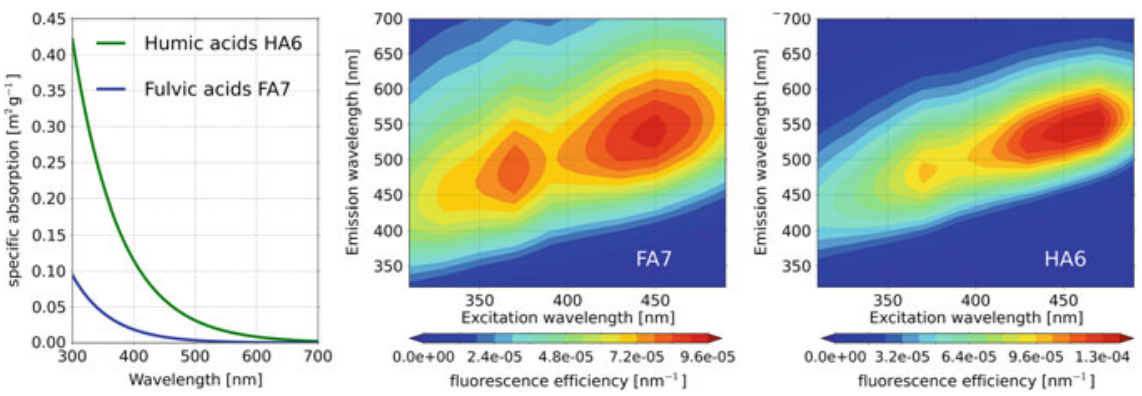

Fig. 1 Specific absorption spectra and fluorescence quantum efficiency functions for samples numbered FA7 (of fulvic acids) and HA6 (of humic acids). Redrawn from Hawes (1992) 
widely applied to excitation-emission matrices (EEMs) and enables qualitative and quantitative characterization of CDOM samples (Ishii and Boyer 2012).

The PARAFAC model is similar to a principal component analysis (PCA), but decomposes the data matrix into a set of trilinear terms and a residual array:

$$
\begin{aligned}
& x_{i j k}=\sum_{f=1}^{F} b_{i f} c_{j f} d_{k f}+\varepsilon_{i j k}, \\
& i=1, \ldots, I ; \quad j=1, \ldots, J ; \quad k=1, \ldots, K
\end{aligned}
$$

According to Stedmon et al. (2003), when applying the PARAFAC model to EEMs, $x_{i j k}$ is the intensity of fluorescence for the $i$ th sample at emission wavelength $j$ and excitation wavelength $k$; $b_{i f}$ is directly proportional to the concentration of the $f$ th component in sample $i ; c_{j f}$ is linearly related to the fluorescence quantum efficiency of the $f$ th component at emission wavelength $j, d_{k f}$ is linearly proportional to the specific absorption coefficient at excitation wavelength $k$. $F$ defines the number of components in the model, and a residual $\varepsilon_{i j k}$ represents the variability not accounted for by the model. The parameters of the PARAFAC model are found by the process of minimizing the sum of squared residuals.

For our study we used the data from a paper by Jørgensen et al. (2011), who conducted a broad analysis of CDOM fluorescence on a global scale and identified seven components with the PARAFAC model, using split half analysis for validation (introduction to the PARAFAC technique and description of its application to CDOM fluorescence are presented in more detail in Stedmon and Bro (2008)). Jørgensen et al. (2011) characterized these components by comparing their spectral characteristics to components identified in earlier studies and pure fluorophores. Spectral characteristics (product of matrices $\mathbf{c}$ and $\mathbf{d}$ ) of seven PARAFAC components are shown in Fig. 2. Jørgensen et al. (2011) identified components 1 and 4 as humic-like DOM. Components 2, 3, 5 and 7 were characterized as amino acidlike DOM, which had spectra similar to phenylalanine, tryptophan and tyrosine. Component 6 had characteristics similar to both groups.

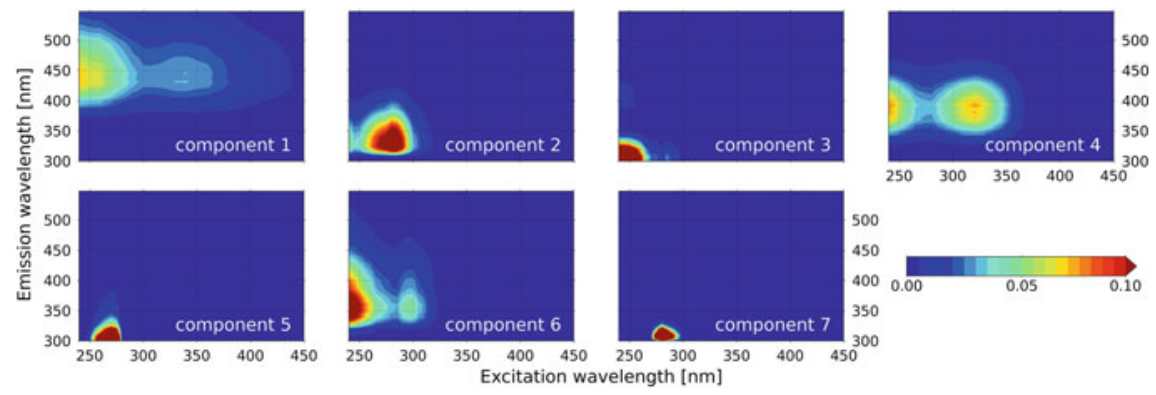

Fig. 2 Fluorescence characteristics of the seven components identified by the PARAFAC analysis and included into radiative transport model SCIATRAN. Redrawn from Jørgensen et al. (2011) 
Because it is not known what the fluorescent components are, the molar absorbance, quantum efficiency and concentration cannot be calculated correctly. Hence, the terms of the PARAFAC model are only proportional to the molar absorbance, quantum efficiency and concentration. We decided to scale the matrices in order to obtain values that are realistic and comparable to the first approach we used for CDOM modeling. Fluorescence quantum yields of CDOM were previously reported to range from 0.005 up to 0.025 (Green and Blough 1994). The values obtained by Hawes (1992), used in the first approach of modeling CDOM fluorescence, vary within these limits (0.009 to 0.019). In order to obtain fluorescence yields of the same order for the PARAFAC components, we scaled matrix $\mathbf{c}$ by a factor of 0.001 , after which the fluorescence yields varied between 0.009 and 0.024 .

The absorption of the PARAFAC components is calculated as a product of matrices $\mathbf{b}$ and $\mathbf{d}$. We decided to scale these matrices so that we obtain absorption similar to the first modeling approach. In case of humic and fulvic acids, we set their concentrations to 1 and $10 \mathrm{~g} \mathrm{~m}^{-3}$, and the sum of $1 \mathrm{~g} \mathrm{~m}^{-3}$ of fulvic and $1 \mathrm{~g} \mathrm{~m}^{-3}$ of humic acids lead to the absorption at $443 \mathrm{~nm}$ of $0.086 \mathrm{~m}^{-1}$. To be consistent, we scaled the product of matrices so that the final absorption of all components at $443 \mathrm{~nm}$ would be similar. In fact, it is only component 1 that absorbs at this wavelength region, and its absorption after applied scaling equaled $0.088 \mathrm{~m}^{-1}$ at $445 \mathrm{~nm}$. The scaled matrices were used to calculate $R$ and then the form of spectral volume inelastic scattering function $\beta$ was derived (Eq. 3). Just like in case of the first modeling approach, we also simulated scenarios for ten times higher concentrations of PARAFAC components. It is important to bear in mind, that we could only scale matrices to realistic values, but their correct values remain unknown.

\section{Results and Discussion}

With the methods described above, the reference spectra were calculated for fulvic and humic acids (according to Hawes 1992) and for seven PARAFAC components identified by Jørgensen et al. (2011). We investigated the spectral characteristics of different CDOM components, particularly in terms of their possible application in the satellite DOAS retrieval technique. In particular, we looked into the narrow sharp features resulting from filling-in of Fraunhofer lines.

We present reference spectra for fulvic and humic acids and PARAFAC components, calculated according to Eq. 2, in Fig. 3a-c. The noticeable sharp and narrow features correspond to filling-in of Franuhofer lines or telluric absorption lines. Among the amino-acid like PARAFAC components (Fig. 3c), fluorescence of component 2 is noticeably stronger than the others at wavelengths above $310 \mathrm{~nm}$. This component was identified as trypthophan-like (Jørgensen et al. 2011). Components 3 and 7 have very weak fluorescence, and component 5 did not produce any fluorescence signal. In general, amino acid-like components have much smaller (up to three orders of magnitude) reference spectra than the humic-like (Fig. 3b). 


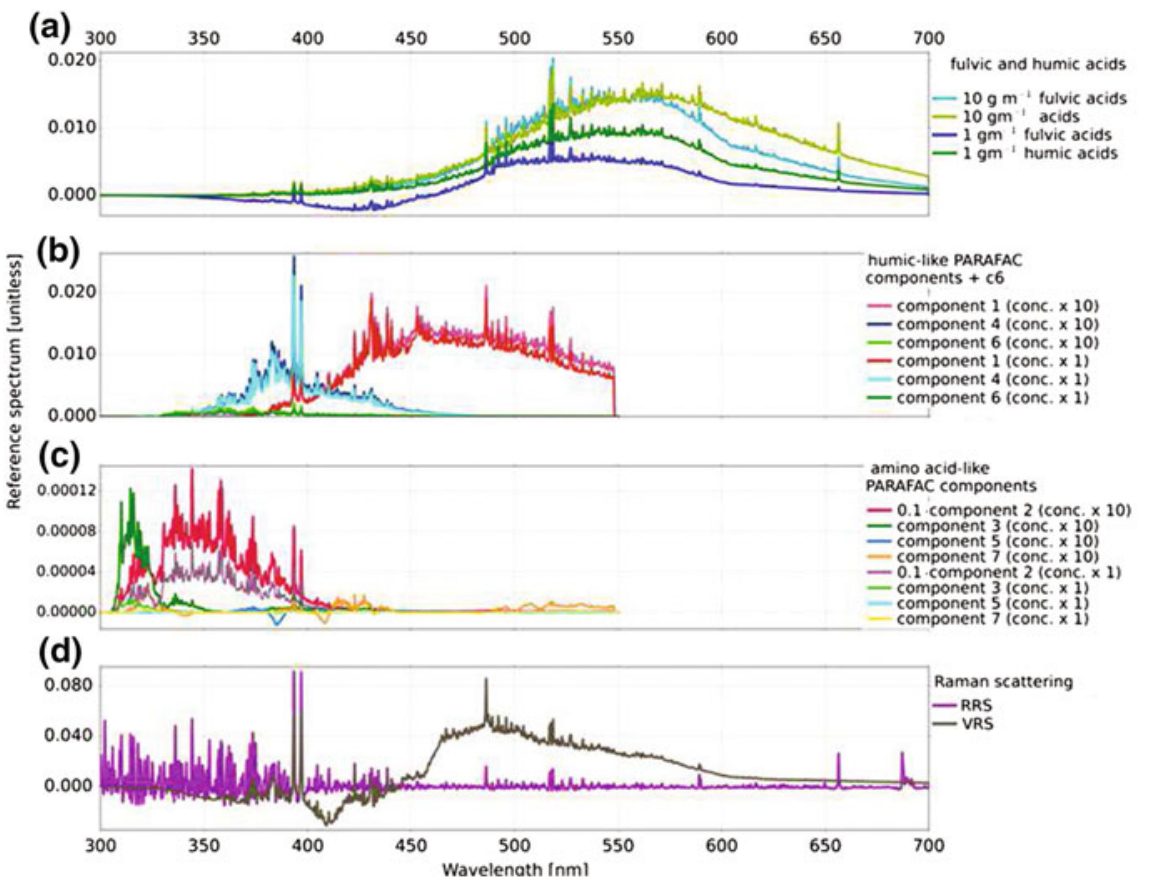

Fig. 3 Calculated reference spectra for: a fulvic and humic acids (Hawes 1992), b, c PARAFAC components (Jørgensen et al. 2011) d RRS and VRS

Amino acid-like components exhibit the fluorescence excitation at shorter wavelengths, usually below $300 \mathrm{~nm}$ (Jørgensen et al. 2011). Due to this, when it is measured with active techniques, the fluorescence signal is strong; however, it is much weaker in natural conditions as there is little electromagnetic radiation reaching the ocean surface in this wavelengths region. One reason is the shape of the solar spectrum, which has its maximum irradiance in longer wavelengths near $500 \mathrm{~nm}$, and is rapidly decreasing towards shorter wavelengths. The second reason is the strong absorption of ozone in the UV region. Humic-like components of CDOM show stronger fluorescence features under natural conditions, as their excitation spectral region is in longer wavelengths, above $300 \mathrm{~nm}$, where more light is available in the water column.

The spectral shape of humic-like PARAFAC components differs significantly from the spectra of humic acids obtained using the Hawes (1992) approach (Fig. 3a). In case of the latter, their fluorescence emissions start in longer wavelengths and reach further up to $700 \mathrm{~nm}$, which is not the case for the PARAFAC measurements. This difference is probably due to the techniques used by Hawes (1992) to separate and measure humic and fulvic acids absorption and fluorescence. It was shown in previous studies that $\mathrm{CDOM}$ extracted with $\mathrm{C}-18$ cartridges (which were used in this case) did not always retain the optical properties of the original 
water sample (e.g. Vodacek 1989). This conclusion is supported by the results of Green and Blough (1994), which indicate that this isolation procedure preferentially extracts longer wavelength absorbing and fluorescing material that also exhibits higher quantum yields. However, EEMs from oligotrophic waters might also underestimate absorption and fluorescence at longer wavelengths. We can also note, that a ten fold increase in the concentration of CDOM leads to a much smaller increase in filling-in of Fraunhofer lines. This is true in case of both modeling approaches.

In the following, the fluorescence reference spectra were compared to the reference spectra for RRS and VRS, which also lead to filling-in of Fraunhofer lines (Grainger and Ring 1962; Kattawar and Xu 1992). These features can be observed in the whole spectral region of the CDOM fluorescence (Fig. 3d). We can detect that RRS produces especially strong signature in the short wavelength region, where the amino acid-like part of DOM produces weak fluorescence. However, we have to bear in mind, that the PARAFAC components were calculated using a scaling introduced by us and hence the values obtained might deviate from the correct values.

In Fig. 4 the differential parts (after subtracting the polynomial in the spectral region of interest) of the reference spectra, calculated according to Eq. 2, for different inelastic processes are shown. Their structures originate from filling-in of two Fraunhofer lines: $\mathrm{Fe}$ I lines at $344 \mathrm{~nm}$ (region of relatively strong fluorescence of amino acid-like DOM) and $\mathrm{H} \beta$ at $486 \mathrm{~nm}$ (the region of fluorescence of humic like $\mathrm{DOM})$. Fraunhofer line $\mathrm{H} \beta$ at $486 \mathrm{~nm}$ also lies close to the CDOM fluorescence maximum reported in previous studies at $490 \mathrm{~nm}$ (e.g. Vodacek et al. 1994; Pozdnyakov et al. 2002). For CDOM spectra we summed up contributions from all defined components of either model. Filling-in of those Fraunhofer lines due to the CDOM fluorescence is relatively weak when compared to filling-in resulting from Raman scattering (RRS, VRS for clear water) for both spectral ranges. For the short-wave fluorescence of amino acid-like components (Fe I lines at $344 \mathrm{~nm}$ ), the strength of RRS is absolutely dominant over all other inelastic processes (Fig. 4, left panel).

In case of longer wavelengths $(\mathrm{H} \beta$ at $486 \mathrm{~nm})$, all processes lead to filling-in of the similar strength (Fig. 4, right panel). In addition, we investigated the effect of
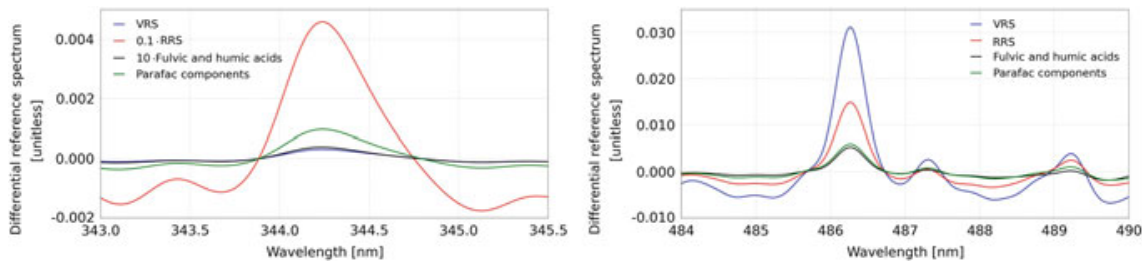

Fig. 4 The differential parts of the reference spectra calculated according to Eq. 2 and Sect. 2.2 for different inelastic processes in the spectral region of Fraunhofer lines Fe I at $344 \mathrm{~nm}$ (left panel) and $\mathrm{H} \beta$ at $486 \mathrm{~nm}$ (right panel) 


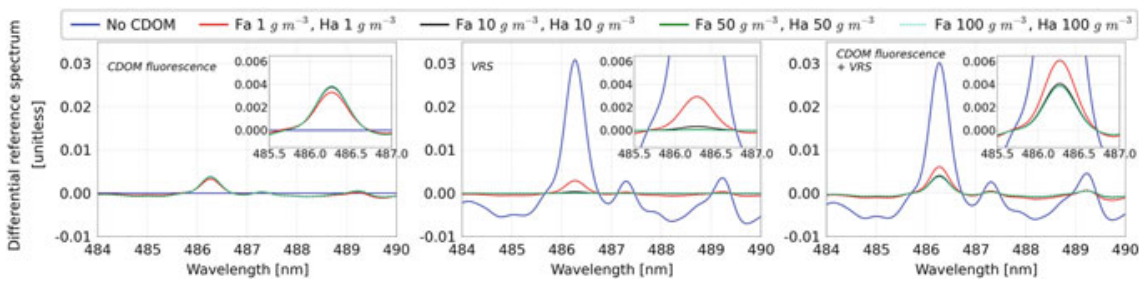

Fig. 5 The differential part of the reference spectra calculated according to Eq. 2 in the spectral region of Fraunhofer line $\mathrm{H} \beta$ at $486 \mathrm{~nm}$ calculated for different concentrations of humic and fulvic acids and for different inelastic processes: CDOM fluorescence (left panel), VRS (middle panel), CDOM fluorescence and VRS simultaneously (right panel)

varying concentration of fulvic and humic acids (from none to $100 \mathrm{~g} \mathrm{~m}^{-3}$ of each) on the differential reference spectra of CDOM and VRS. In this wavelength region it is more likely to observe the CDOM fluorescence with satellite measurements. However, in clear waters, the VRS signal is dominant, but is weakening with increasing CDOM concentrations (Fig. 5 middle panel). In case of CDOM concentration of $1 \mathrm{~g} \mathrm{~m}^{-3}$ fulvic and $1 \mathrm{~g} \mathrm{~m}^{-3}$ humic acids, both CDOM fluorescence and VRS lead to similar filling-in. In case of CDOM being a mixture of $50 \mathrm{~g} \mathrm{~m}^{-3}$ of fulvic and $50 \mathrm{~g} \mathrm{~m}^{-3}$ humic acids, VRS signal is one order of magnitude weaker than CDOM fluorescence, and for $100 \mathrm{~g} \mathrm{~m}^{-3}$ of fulvic and $100 \mathrm{~g} \mathrm{~m}^{-3}$ of humic acids, the VRS signal is negligible (Fig. 5). However, the filling-in due to CDOM fluorescence itself remains almost constant through all the scenarios of different CDOM concentrations (Fig. 5 left panel). It is due to the fact that with increasing CDOM concentration, light is absorbed stronger and does not penetrate that deep into the water column, hence the bulk CDOM fluorescence signal originates from thinner surface layer of the ocean and is not significantly higher. Our results agree with the previous studies by Pozdnyakov et al. (2002), who showed that VRS is negligible for waters with high CDOM concentrations. Their results also indicate that the CDOM fluorescence signal does not increase substantially with much higher CDOM concentrations, even though it becomes relatively more significant, as in general the upwelling radiance decreases. However, contrary to their results, we found that the VRS in clear waters is stronger than CDOM fluorescence in waters of high CDOM concentration. These discrepancies might be due to the fact that while we are investigating the impact of inelastic process on the filling-in of Fraunhofer lines at the top of the atmosphere, Pozdnyakov et al. (2002), focused on the water volume reflectance. Furthermore, we used the coupled ocean-atmosphere radiative transfer model, while Pozdnyakov et al. (2002), used simple hydrooptical models, which calculate the volume reflectance arising from CDOM fluorescence directly from optical properties of water and CDOM. For scenarios of simultaneous CDOM fluorescence and VRS, we observe that the combined filling-in signal is in fact getting weaker with increasing CDOM concentration (Fig. 5 right panel).

Hence, filling-in signals due to CDOM fluorescence and VRS are coupled: when there is no CDOM, the VRS signal is strong. On the other hand, when CDOM 
concentrations are high and VRS signal is very weak, there is still significant CDOM fluorescence signal. It follows, that the filling-in signal can be due to strong CDOM fluorescence or strong VRS. However, the VRS is in general a stronger process and filling-in originating from CDOM fluorescence does not increase strongly with increasing CDOM concentration, so in the regions of high CDOM we see in fact weaker filling-in due to absorption of CDOM and weakening of VRS.

As an example, we have run two simple DOAS retrievals for June and July 2009 on the SCIAMACHY (Bovensmann et al. 1999) data in the spectral regions of those Fraunhofer lines (343-345.5 nm and 484-490 nm). For each retrieval we fitted only one reference spectrum, which was a sum of reference spectra of all the PARAFAC components for a respective spectral window. In fact, due to spectral similarities of the filling-in of Fraunhofer lines caused by different processes, the fitted spectra correspond to the contribution from all inelastic processes. We focused on the Atlantic area close to the river mouth of the Amazon, where loading of the terrestrial CDOM into the ocean is generally very high, and where the highest CDOM absorption in the surface waters of open ocean was found in a study by Nelson and Siegel (2013). In Fig. 6 results of fit factors for the two spectral windows are compared to the MODIS-Aqua absorption coefficient for dissolved and detrital particulate matter at $443 \mathrm{~nm}$ derived from the Garver-Siegel-Maritorena algorithm (Garver and Siegel 1997; Maritorena et al. 2002). In case of using the filling-in of $\mathrm{H} \beta$ line in the retrieval (Fig. 6b), in the area of high CDOM concentration, where we expect strong CDOM fluorescence, the retrieved filling-in of Fraunhofer lines is in fact weaker. It is due to weaker VRS, as described above. For the CDOM fluorescence retrieval in shorter wavelengths (Fig. 6a), when preliminarily examined, the spatial structure of the Amazon plum is not seen and the results are completely dominated by RRS (which is stronger towards higher solar zeniths angle hence higher latitudes). However, these results do not include correction for Raman scattering contribution to filling-in of Fraunhofer lines, which would be necessary for a solid comparison.

(a)

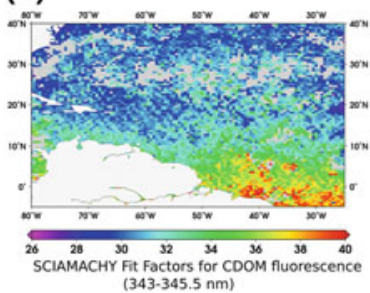

(b)

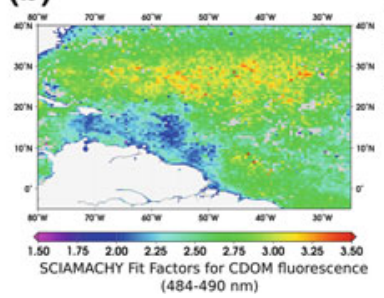

(c)

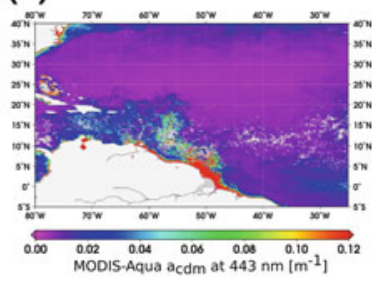

Fig. 6 Maps of retrieved CDOM fluorescence fit factors for 2 months (June and July 2009) in two different spectral regions (a, b) compared with MODIS-Aqua absorption coefficient for dissolved and detrital particulate matter $a_{c d m}$ at $443 \mathrm{~nm}\left[\mathrm{~m}^{-1}\right]$ (c) 


\section{Conclusions and Outlook}

Our study shows that although it was possible to estimate inelastic processes with the DOAS method (Vountas et al. 1998, 2003, 2007), it is difficult to use this approach to retrieve CDOM fluorescence from hyperspectral satellite data, mainly because of dominant effects of other inelastic processes (RRS, VRS). However, our modeling studies prove that for coastal areas, where VRS is weak, fluorescence of humic-like CDOM is a significant source of filling-in. Hence, selection of waters with high CDOM concentration should be further studied with satellite or airborne sensor providing much smaller footprints, as well as the good choice of wavelength regions. It is even more difficult to study the composition of CDOM and retrieve fluorescence for the amino acid-like components of DOM, which have excitation and emission in UV-wavelengths. It is due to stronger RRS for shorter wavelengths, very low electromagnetic radiation reaching the surface in the excitation wavelengths, and similar effects in filling-in of Fraunhofer lines. In addition to looking at filling-in of individual Fraunhofer lines, it could be possible to compare broader structures, as filling-in spectra of different CDOM components have spectra distinct from RRS and VRS (Fig. 3). However, due to various composition of CDOM worldwide, those structures vary significantly in time and space, and are not known a priori. Additional precise EEM measurements accompanied by correctly calculated molar absorbance, quantum efficiency and concentration, are needed for improving the modeling of CDOM fluorescence. Combined use of narrow and broad fluorescence features and an appropriate method to account for inelastic processes are as well necessary for further investigating the retrieval of CDOM fluorescence.

Acknowledgments Aleksandra Wolanin gratefully acknowledges financial support provided by the Earth System Science Research School (ESSReS), an initiative of the Helmholtz Association of German Research Centres (HGF) at the Alfred Wegener Institute for Polar and Marine Research (AWI) and HGF Innovative Network Fund (PHYTOOPTICS project). Authors thank DLR and ESA for SCIAMACHY level-1 data. We also acknowledge the MODIS mission scientists and associated NASA personnel for the production of the data used in this research effort. Analyses and visualizations were produced with the Giovanni online data system, developed and maintained by the NASA GES DISC. We also thank C. Stedmon and K. Carder for providing CDOM data and their helpful comments throughout the development of this chapter.

\section{References}

Babin M (2003) Variations in the light absorption coefficients of phytoplankton, nonalgal particles, and dissolved organic matter in coastal waters around Europe. J Geophys Res 108(C7)

Blum M, Rozanov V, Burrows J, Bracher A (2012) Coupled ocean-atmosphere radiative transfer model in the framework of software package SCIATRAN: selected comparisons to model and satellite data. Adv Space Res 49(12):1728-1742

Bovensmann H, Burrows JP, Buchwitz M, Frerick J, Noël S, Rozanov VV, Chance KV, Goede APH (1999) SCIAMACHY: mission objectives and measurement modes. J Atmos Sci 56 (2): $127-150$ 
Bracher A, Vountas M, Dinter T, Burrows J, Röttgers R, Peeken I (2009) Quantitative observation of cyanobacteria and diatoms from space using PhytoDOAS on SCIAMACHY data. Biogeosciences 6:751-764

Burrows JP, Weber M, Buchwitz M, Rozanov V, Ladstätter-Weißenmayer A, Richter A, DeBeek R, Hoogen R, Bramstedt K, Eichmann K-U (1999) The global ozone monitoring experiment (GOME): mission concept and first scientific results. J Atmos Sci 56(2):151-175

Garver SA, Siegel DA (1997) Inherent optical property inversion of ocean color spectra and its biogeochemical interpretation: 1. time series from the Sargasso Sea. J Geophys Res 102 (C8): 18607

Grainger JF, Ring J (1962) Anomalous Fraunhofer line profiles. Nature 193(4817):762

Green SA, Blough NV (1994) Optical absorption and fluorescence properties of chromophoric dissolved organic matter in natural waters. Limnol Oceanogr 39(8):1903-1916

Hawes SK (1992) Quantum fluorescence efficiencies of marine fulvic and humic acids. M.Sc. thesis. University of South Florida, Tampa

Hudson N, Baker A, Reynolds D (2007) Fluorescence analysis of dissolved organic matter in natural, waste and polluted waters-a review. River Res Appl 23(6):631-649

Ishii SKL, Boyer TH (2012) Behavior of reoccurring PARAFAC components in fluorescent dissolved organic matter in natural and engineered systems: a critical review. Environ Sci Technol 46(4):2006-2017

Jørgensen L, Stedmon CA, Kragh T, Markager S, Middelboe M, Søndergaard M (2011) Global trends in the fluorescence characteristics and distribution of marine dissolved organic matter. Mar Chem 126(1-4):139-148

Kattawar GW, Xu X (1992) Filling in of Fraunhofer lines in the ocean by Raman scattering. Appl Opt 31(30):6491

Maritorena S, Siegel D, Peterson A (2002) Optimization of a semianalytical ocean color model for global-scale applications. Appl Opt 41(15):2705-2714

Mobley CD (1994) Light and water: radiative transfer in natural waters. Academic Press, San Diego (u.a.)

Morel A, Gentili B (2009) A simple band ratio technique to quantify the colored dissolved and detrital organic material from ocean color remotely sensed data. Remote Sens Environ 113 (5):998-1011

Nelson NB, Siegel DA (2013) The global distribution and dynamics of chromophoric dissolved organic matter. Annu Rev Mar Sci 5(1):447-476

Pozdnyakov D, Grassl H, Lyaskovsky A, Pettersson L (2002) Numerical modelling of transspectral processes in natural waters: Implications for remote sensing. Int J Remote Sens 23(8):1581-1607

Richter A, Burrows JP, Nüß H, Granier C, Niemeier U (2005) Increase in tropospheric nitrogen dioxide over China observed from space. Nature 437(7055):129-132

Rozanov V, Rozanov A, Kokhanovsky A, Burrows J (2014) Radiative transfer through terrestrial atmosphere and ocean: software package SCIATRAN. J Quant Spectrosc Radiat Transfer 133:13-71

Rozanov VV, Rozanov AV (2010) Differential optical absorption spectroscopy (DOAS) and air mass factor concept for a multiply scattering vertically inhomogeneous medium: theoretical consideration. Atmos Meas Tech 3:751-780

Sadeghi A, Dinter T, Vountas M, Taylor B, Altenburg-Soppa M, Bracher A (2012) Remote sensing of coccolithophore blooms in selected oceanic regions using the PhytoDOAS method applied to hyper-spectral satellite data. Biogeosciences 9:2127-2143

Sinnhuber B-M, Sheode N, Sinnhuber M, Chipperfield MP, Feng W (2009) The contribution of anthropogenic bromine emissions to past stratospheric ozone trends: a modelling study. Atmos Chem Phys 9:2863-2871

Stedmon C, Markager S, Bro R (2003) Tracing dissolved organic matter in aquatic environments using a new approach to fluorescence spectroscopy. Mar Chem 82(3-4):239-254

Stedmon CA, Bro R (2008) Characterizing dissolved organic matter fluorescence with parallel factor analysis: a tutorial. Limnol Oceanogr Methods 6:572-579 
Vodacek A (1989) Synchronous fluorescence spectroscopy of dissolved organic matter in surface waters: application to airborne remote sensing. Remote Sens Environ 30(3):239-247

Vodacek A, Green SA, Blough NV (1994) An experimental model of the solar-stimulated fluorescence of chromophoric dissolved organic matter. Oceanography 39(1)

Vountas M, Dinter T, Bracher A, Burrows JP, Sierk B (2007) Spectral studies of ocean water with space-borne sensor SCIAMACHY using differential optical absorption spectroscopy (DOAS). Ocean Sci 3(3):429-440

Vountas M, Richter A, Wittrock F, Burrows JP (2003) Inelastic scattering in ocean water and its impact on trace gas retrievals from satellite data. Atmos Chem Phys 3(5):1365-1375

Vountas M, Rozanov V, Burrows J (1998) Ring effect: impact of rotational Raman scattering on radiative transfer in Earth's atmosphere. J Quant Spectrosc Radiat Transfer 60(6):943-961 